\title{
CLASSICAL HADRODYNAMICS: A NEW APPROACH TO ULTRARELATIVISTIC HEAVY-ION COLLISIONS
}

\author{
Brian W. Bush and J. Rayford Nix \\ Theoretical Division, Los Alamos National Laboratory \\ Los Alamos, New Mexico 87545, USA
}

\begin{abstract}
We discuss a new approach to ultrarelativistic heavy-ion collisions based on classical hadrodynamics for extended nucleons, corresponding to nucleons of finite size interacting with massive meson fields. This new theory provides a natural covariant microscopic approach that includes automatically spacetime nonlocality and retardation, nonequilibrium phenomena, interactions among all nucleons and particle production. In the current version of our theory, we consider $N$ extended unexcited nucleons interacting with massive neutral scalar $(\sigma)$ and neutral vector $(\omega)$ meson fields. The resulting classical relativistic many-body equations of motion are solved numerically without further approximation for soft nucleon-nucleon collisions at $p_{\text {lab }}=14.6,30,60,100$ and $200 \mathrm{GeV} / c$ to yield the transverse momentum imparted to the nucleons. For the future development of the theory, the isovector pseudoscalar $\left(\pi^{+}, \pi^{-}, \pi^{0}\right)$, isovector scalar $\left(\delta^{+}, \delta^{-}, \delta^{0}\right)$, isovector vector $\left(\rho^{+}, \rho^{-}, \rho^{0}\right)$ and neutral pseudoscalar $(\eta)$ meson fields that are known to be important from nucleon-nucleon scattering experiments should be incorporated. In addition, the effects of quantum uncertainty on the equations of motion should be included by use of techniques analogous to those used by Moniz and Sharp for nonrelativistic quantum electrodynamics.
\end{abstract}

\section{Introduction}

Many of you are involved in the search for the quark-gluon plasma - a predicted new phase of nuclear matter where quarks roam almost freely throughout the medium instead of being confined to individual nucleons. Experimental identification of the quark-gluon plasma will require accurate predictions for ultrarelativistic heavy-ion collisions on the basis of conventional nuclear physics, with the known hadronic degrees of freedom properly taken into account. Significant deviations between these predictions and experimental data would then signal the onset of new phenomena such as a quark-gluon plasma.

With this goal in mind, we have developed at Los Alamos an entirely new microscopic many-body approach to ultrarelativistic heavy-ion collisions based on classical hadrodynamics for extended nucleons, corresponding to nucleons of finite size interacting with massive meson fields. This approach, which satisfies a priori the physical conditions that exist at ultrarelativistic energies, is manifestly Lorentz-covariant and allows for nonequilibrium phenomena, interactions among all nucleons and particle production.

The physical input underlying this approach consists of Lorentz invariance (which includes energy and momentum conservation), nucleons of finite size interacting with 
massive meson fields and the classical approximation applied in domains where it should be reasonably valid. This starting point builds upon the traditional hadronic description of nuclear processes that has in the past been so successful in a wide variety of situations. By treating the underlying quarks and gluons implicitly in terms of nucleons and mesons, we are able to solve the resulting classical relativistic many-body equations of motion numerically without further approximation. In particular, we do not need to make either a mean-field approximation, a perturbative expansion in coupling strength or a superposition of two-body collisions.

The motivation for the classical approximation in this new theory is that at bombarding energies of many $\mathrm{GeV}$ per nucleon, the de Broglie wavelength of projectile nucleons is extremely small compared to all other length scales in the problem. In addition, the Compton wavelength of the nucleon is small compared to its radius, so that effects due to the intrinsic size of the nucleon dominate those due to quantum uncertainty. Finally, the angular momentum is typically several hundred $\hbar$ and the radiated energy corresponds to several meson masses. The classical approximation for nucleon trajectories should therefore be valid, provided that the effect of the finite nucleon size on the equations of motion is taken into account.

We describe in sect. 2 the present version of our theory, which includes the neutral scalar $(\sigma)$ and neutral vector $(\omega)$ meson fields. This permits a qualitative discussion of such physically relevant points as the effect of the finite nucleon size on the equations of motion, an inherent spacetime nonlocality that may be responsible for significant collective effects and particle production through massive bremsstrahlung. The $\sigma$ and $\omega$ mesons that are produced through this mechanism will subsequently decay primarily into pions with some photons also. The resulting classical relativistic equations of motion are solved in sect. 3 for soft nucleon-nucleon collisions at $p_{\text {lab }}=14.6,30,60,100$ and $200 \mathrm{GeV} / c$ to yield the transverse momentum imparted to the nucleons. Section 4 discusses future directions for the sustematic development of the theory. Further details are given in a series of papers, 10 although not all of the equations appearing in some of the earlier publications are in their final form.

\section{Equations of motion}

Our action for $N$ extended unexcited nucleons interacting with massive scalar and vector meson fields is

$$
\begin{aligned}
I= & \overbrace{-M_{0} \sum_{i=1}^{N} \int d \tau_{i} \sqrt{\dot{q}_{i}^{2}}}^{\text {Nucleons }}+\overbrace{\frac{1}{8 \pi} \int d^{4} x\left[(\partial \phi)^{2}-m_{\mathrm{s}}^{2} \phi^{2}\right]}^{\text {Scalar field }} \\
& -\underbrace{\frac{1}{8 \pi} \int d^{4} x\left(\frac{1}{2} G^{2}-m_{\mathrm{v}}{ }^{2} V^{2}\right)}_{\text {Vector field }}-\underbrace{\int d^{4} x(j \phi+K \cdot V)}_{\text {Interaction }},
\end{aligned}
$$

where $M_{0}$ is the bare nucleon mass and $q_{i}$ is the four-position of the $i$ th nucleon, whose trajectory is given by $q_{i}=q_{i}\left(\tau_{i}\right)$. A dot represents the derivative with respect to $\tau_{i}$. In the action the four-velocities are not constrained so that $\dot{q}_{i}^{2}=1$ and $\tau_{i}$ is not yet identified as the proper time; it is only in the equations of motion, which are derived as a result of the variation of $I$, that this is true. We use the metric $g^{\mu \nu}=\operatorname{diag}(1,-1,-1,-1)$, write 
four-vectors as $q^{\mu}=\left(q^{0}, \mathbf{q}\right)=\left(q^{t}, q^{x}, q^{y}, q^{z}\right)$ and use units in which $\hbar=c=1$. The scalar potential is denoted by $\phi$, the four-vector potential by $V$ and the meson masses by $m_{\mathrm{s}, \mathrm{v}}$. The vector field strength tensor is

$$
G^{\mu \nu}=\partial^{\mu} V^{\nu}-\partial^{\nu} V^{\mu}
$$

the scalar source density is

$$
j(x)=g_{\mathrm{s}} \sum_{i=1}^{N} \int d \tau_{i} \rho\left(x-q_{i}, \dot{q}_{i}\right) \sqrt{\dot{q}_{i}^{2}}
$$

and the vector source density is

$$
K^{\mu}(x)=g_{\mathrm{v}} \sum_{i=1}^{N} \int d \tau_{i} \rho\left(x-q_{i}, \dot{q}_{i}\right) \dot{q}_{i}^{\mu},
$$

where $\rho$ is the four-dimensional mass density of the nucleon, the spatial part of which we assume to be exponential in the nucleon's rest frame. The values 11 13 that we are currently using for the six physical constants appearing in our theory are nucleon mass $M=938.91897 \mathrm{MeV}$, scalar $(\sigma)$ meson mass $m_{\mathrm{s}}=550 \mathrm{MeV}$, vector $(\omega)$ meson mass $m_{\mathrm{v}}=781.95 \mathrm{MeV}$, scalar interaction strength $g_{\mathrm{s}}^{2}=7.29$, vector interaction strength $g_{\mathrm{v}}{ }^{2}=10.81$ and nucleon r.m.s. radius $R_{\mathrm{rms}}=0.862 \mathrm{fm}$.

In ref. 7 we have derived exact equations of motion for the above action in two limits: (1) relativistic point nucleons and (2) nonrelativistic extended nucleons. We then generalize covariantly to obtain relativistic equations of motion for extended nucleons, which can be written as

$$
M_{i}^{*} a_{i}^{\mu}=f_{\mathrm{s}, i}^{\mu}+f_{\mathrm{v}, i}^{\mu}+f_{\mathrm{s}, \mathrm{ext}, i}^{\mu}+f_{\mathrm{v}, \mathrm{ext}, i}^{\mu},
$$

where $M_{i}^{*}$ is the effective mass, $f_{\mathrm{s}, i}^{\mu}$ is the scalar self-force, $f_{\mathrm{v}, i}^{\mu}$ is the vector self-force, $f_{\mathrm{s}, \mathrm{ext}, i}^{\mu}$ is the scalar external force and $f_{\mathrm{v}, \mathrm{ext}, i}^{\mu}$ is the vector external force. These equations of motion are second-order, nonlinear, integrodifferential equations with four dimensions per particle.

\section{Transverse momentum for soft nucleon-nucleon collisions}

To solve our equations of motion (5) we use a fourth-order Adams-Moulton predictorcorrector algorithm with adaptive step sizes. The integrations over proper time are done with a special error-minimizing application of Lagrange's four-point (cubic) interpolation formulas. We present here results on the transverse momentum imparted in the soft collision of two nucleons at laboratory momentum $p_{\text {lab }}=14.6,30,60,100$ and $200 \mathrm{GeV} / c$. At three of these momenta substantial experimental data exist for heavy-ion collisions 14 , and at the remaining two momenta experimental data exist for proton-proton collisions. 16 Results calculated for the scattering angle, radiated energy and rapidity are described in ref. 8.

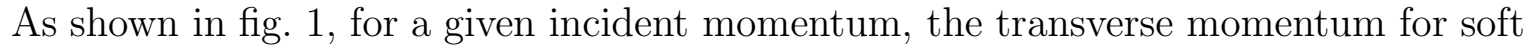
reactions has a maximum value at a certain impact parameter and decreases to zero for both head-on and distant collisions. The maximum transverse momentum increases slowly with increasing incident momentum in this range, and the impact parameter at which 
Fig. 1: Calculated dependence of the transverse momentum upon impact parameter for soft nucleonnucleon collisions at five incident laboratory momenta.

this maximum occurs decreases. For ultrarelativistic collisions this impact parameter is approximately the distance at which the transversely dominating static vector force for extended nucleons 6 has its maximum. At the other extreme of low incident momentum, the opposing scalar and vector forces are of similar magnitude and give rise for small impact parameter to the more complicated behaviour of the double-dot-dashed curve in fig. 11.

The qualitative behaviour of our results can be understood in terms of the nature of the external forces. The repulsive vector force scales as the Lorentz factor $\gamma$ in both the longitudinal and transverse directions, whereas the attractive scalar force scales as $\gamma^{2}$ in the longitudinal direction and as unity in the transverse direction. This implies that the vector force will dominate the transverse acceleration and the scalar force will dominate the longitudinal acceleration. For a given impact parameter the scattering angle and transverse momentum will be essentially proportional to the vector interaction strength $g_{\mathrm{v}}{ }^{2}$, and the radiated energy will be essentially proportional to $\gamma$ times the scalar interaction strength $g_{\mathrm{s}}^{2}$.

As an initial test of the theory to describe the gross features of soft nucleon-nucleon collisions, we compare in fig. 2 experimental and theoretical values of the mean transverse momentum $\left\langle p_{\mathrm{T}}\right\rangle$ as a function of total centre-of-mass energy $\sqrt{s}$. Because the classical total cross section diverges, it is necessary when calculating such average quantities to select a particular cutoff in impact parameter, or equivalently, a value of the total cross section, for which purpose we have used $122 \sigma_{\text {tot }}=40 \mathrm{mb}$. Our results calculated with the values of the constants listed in sect. 2 are given by the solid curve in fig. 2 and are seen to be both lower in magnitude and to increase more rapidly with $\sqrt{s}$ than the experimental values.17 18 However, the theoretical curve is calculated for all values of rapidity corresponding to a $40 \mathrm{mb}$ total cross section, whereas the experimental results are peak values in the central region, where there is a short plateau in rapidity. Furthermore, the experimental values include high- $p_{\mathrm{T}}$ contributions arising from hard collisions. Because 
Fig. 2: Comparison of experimental and theoretical values of the mean transverse momentum $\left\langle p_{\mathrm{T}}\right\rangle$ as a function of total centre-of-mass energy $\sqrt{s}$.

of the rough proportionality of $\left\langle p_{\mathrm{T}}\right\rangle$ to the vector interaction strength $g_{\mathrm{v}}{ }^{2}$, we show with the dotted curve in fig. 2 a simple estimate obtained by multiplying the solid curve by the ratio of $g_{\mathrm{v}}{ }^{2}=17.26$ obtained by Bryan and Scott 19 from an analysis of nucleon-nucleon scattering at laboratory kinetic energies between 0 and $350 \mathrm{MeV}$ to the value appearing in sect. 2.

\section{Future directions}

From nucleon-nucleon scattering experiments we know that several additional meson fields are important and must be included for a quantitative description:20

- Isovector pseudoscalar $\left(\pi^{+}, \pi^{-}, \pi^{0}\right)$

- Isovector scalar $\left(\delta^{+}, \delta^{-}, \delta^{0}\right)$

- Isovector vector $\left(\rho^{+}, \rho^{-}, \rho^{0}\right)$

- Neutral pseudoscalar $(\eta)$

The next step in the systematic development of the theory should be the inclusion of these additional meson fields. In addition, the effects of quantum uncertainty on the equations of motion should be studied and included if they are important. This should be possible by use of techniques analogous to those used by Moniz and Sharp for nonrelativistic quantum electrodynamics.21

Once these two formal items are completed, the full version of the theory should be used to calculate ultrarelativistic heavy-ion reactions that are being studied experimentally. Systematic comparisons between the calculated and experimental results for such quantities as differential cross sections, transverse momentum distributions, particle multiplicity distributions, rapidity distributions and particle-particle correlations will enable us to decide the usefulness of classical hadrodynamics for understanding these phenomena.

This work was supported by the U. S. Department of Energy. 


\section{References}

[1] A. J. Sierk, R. J. Hughes and J. R. Nix, Proc. 6th Winter Workshop on nuclear dynamics, Jackson Hole, Wyoming, 1990, Lawrence Berkeley Laboratory report LBL28709 (1990) p. 119

[2] A. J. Sierk, R. J. Hughes and J. R. Nix, Contributed Papers, Symp. in honor of Akito Arima: nuclear physics in the 1990's, Santa Fe, New Mexico, 1990, report (1990) p. 118

[3] B. W. Bush, J. R. Nix and A. J. Sierk, Advances in nuclear dynamics, Proc. 7th Winter Workshop on nuclear dynamics, Key West, Florida, 1991 (World Scientific, Singapore, 1991) p. 282

[4] B. W. Bush, J. R. Nix and A. J. Sierk, Proc. 4th Conf. on the intersections between particle and nuclear physics, Tucson, Arizona, 1991, AIP Conference Proceedings 243 (American Institute of Physics, New York, 1992) p. 835

[5] B. W. Bush and J. R. Nix, Contributed Papers and Abstracts, Quark matter '91, Ninth Int. Conf. on ultra-relativistic nucleus-nucleus collisions, Gatlinburg, Tennessee, 1991, report (1991) p. T98

[6] B. W. Bush and J. R. Nix, Advances in nuclear dynamics, Proc. 8th Winter Workshop on nuclear dynamics, Jackson Hole, Wyoming, 1992 (World Scientific, Singapore, 1992) p. 311

[7] B. W. Bush and J. R. Nix, Ann. of Phys. 227 (1993) 97

[8] B. W. Bush and J. R. Nix, Nucl. Phys. A560 (1993) 586

[9] J. R. Nix, Advances in nuclear dynamics, Proc. 10th Winter Workshop on nuclear dynamics, Snowbird, Utah, 1994 (World Scientific, Singapore, 1994) to be published

[10] B. W. Bush and J. R. Nix, Proc. 7th Int. Conf. on nuclear reaction mechanisms, Varenna, Italy, 1994, to be published

[11] B. D. Serot and J. D. Walecka, Adv. Nucl. Phys. 16 (1986) 1

[12] K. Hikasa et al., Phys. Rev. D45 (1992) S1

[13] B. D. Serot, Phys. Lett. 68B (1979) 146

[14] Quark Matter '91, Proc. Ninth Int. Conf. on ultra-relativistic nucleus-nucleus collisions, Gatlinburg, Tennessee, 1991, Nucl. Phys. A544 (1992) 1c

[15] M. J. Tannenbaum, Int. J. Mod. Phys. A4 (1989) 3377

[16] G. P. Yost et al., Lawrence Berkeley Laboratory report LBL-90 revised (1986)

[17] G. J. Alner et al., Phys. Rep. 154 (1987) 247

[18] W. Busza and R. Ledoux, Ann. Rev. Nucl. Part. Sci. 38 (1988) 119

[19] R. Bryan and B. L. Scott, Phys. Rev. 177 (1969) 1435

[20] R. Machleidt, Adv. Nucl. Phys. 19 (1989) 189

[21] E. J. Moniz and D. H. Sharp, Phys. Rev. D15 (1977) 2850 